\title{
Reprint
}

\section{Relative Direction Determination in Mobile Computing Networks}

\author{
A. Kalis and T. Antonakopoulos
}

The IEEE Instrumentation and Measurement Technology Conference - IMTC/2001

BUDAPEST, HUNGARY, MAY 2001

Copyright Notice: This material is presented to ensure timely dissemination of scholarly and technical work. Copyright and all rights therein are retained by authors or by other copyright holders. All persons copying this information are expected to adhere to the terms and constraints invoked by each author's copyright. In most cases, these works may not be reposted or mass reproduced without the explicit permission of the copyright holder. 


\title{
Relative Direction Determination in Mobile Computing Networks
}

\author{
Antonis Kalis ${ }^{1}$ and Theodore Antonakopoulos ${ }^{2}$ \\ ${ }^{1}$ Computers Technology Institute, Riga Fereou 61, 26221 Patras, Greece \\ ${ }^{2}$ University of Patras, Department of Electrical Engineering, 26500 Rio-Patras, Greece \\ Phone: +30 (61) 997341, Fax: +30 (61) 997341, e-mail: Antonis.Kalis@cti.gr, web: http://www.cti.gr
}

\begin{abstract}
A novel relative direction determination method is presented for stations of mobile computing networks. The method uses a switched beam array for determining the direction of arrival (DOA) of the incident electromagnetic field in a time efficient way, in order to confront the strict timing requirements of packet switching networks. Additionally, the method associates certain MAC layer functions with different radiation patterns of the switched antenna array, in order to determine the orientation of directional beams on both sides of a communication link. The application of the method to an IEEE802.11 wireless network is presented and it is shown how the use of the method optimizes the network performance without requiring any modifications to the existing MAC protocol.
\end{abstract}

\section{INTRODUCTION}

Home networking, mobile computing and small-office/homeoffice (SOHO) multimedia are technological areas with a fast rising demand for extended wireless bandwidth. The wireless channel imposes several restrictions to this demand, due to the effect of multipath fading. One of the most efficient ways to confront the effect of multipath is by using directive antennas on both sides of the wireless link [1]. A directive antenna on the receiver side attenuates the multipath instances arriving from different directions and thus amplifies the signal coming from the transmitter's line of sight (LOS). Moreover, by using a directional antenna on the transmitter, most of the electromagnetic energy of the transmitted signal is directed to the receiver's site, thus minimizing the spurious reflections and diffractions of the signal, that cause multipath fading.

Therefore, optimum transmission performance over a wireless channel is achieved when both communicating entities can determine the relative location of each other and turn their directional beam antennas accordingly. In a static network, where none of the stations is moving, this can be planned beforehand and established permanently. In wireless data networks, the stations' mobility increases the complexity of the network, since moving stations should constantly acquire and retain relative direction information of other mobile stations. In mobile applications that have continuous data transmissions in both directions, as in cell phone applications, relative direction information may be acquired continuously and constantly by integrating a radio direction finding (RDF) algorithm in the physical layer, with the use of phased arrays or switched beam arrays [2]. In mobile computing networks, where there is no continuously established link between transmitter and receiver, relative direction information is hard to acquire and retain. While a receiving station can determine the relative direction of the transmitting station, the transmitter is unable to do so during transmission, since the flow of information is neither continuous nor full duplex. In such networks, pure physical layer functions that determine the direction of arrival (DOA) of the incoming signals are not adequate for determining the correct orientation of the directive beams on both sides of a communication link. A straightforward way to achieve this would be to permit stations to exchange relative direction information, but in this case the MAC layer of the protocol should be altered and the solution would require a significant amount of network bandwidth.

In this paper we propose a method that uses both physical layer and MAC layer functions for acquiring relative direction information on both sides of a packet switched communication link. On the receiver's end the proposed method is based on a RDF algorithm and its association with switched beam arrays. The switched arrays are preferred for mobile computing network applications compared to phased arrays, since they satisfy the cost, size and power consumption restrictions of mobile terminals. RDF algorithms for switched beam arrays have already been reported [3], but they target cell phone applications with less strict timing requirements than packet switching networks. A modified version of such an algorithm is adopted and integrated in the proposed method, in order to meet the MAC protocol timing restrictions. On the other hand, in order to provide a transmitting station with information regarding the relative direction of a receiver, the method associates some MAC layer frames with certain radiation patterns of the array, without altering the MAC protocol functionality. The proposed method focuses on the CSMA/CA protocol and is developed on an IEEE802.11 platform.

Section II describes the direction finding method that is used on the area of DOA determination using switched beam arrays. Such a switched beam array, suitable for the implementation of this method, is also presented in this section. Section III describes the relative direction determination method in an IEEE802.11 mobile computing 


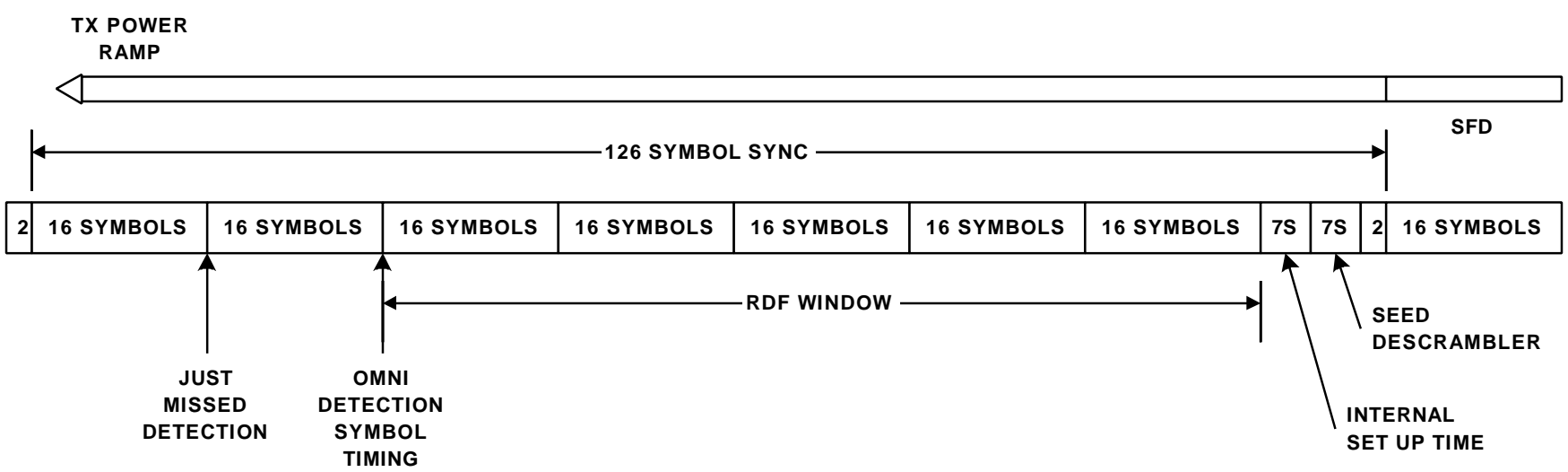

Fig. 1: The preamble period of a IEEE802.11 DSSS physical layer header

network. Simulated results show how the proposed method optimizes the overall performance of the network, without changing the MAC layer functionality.

\section{DIRECTION FINDING}

In packet switching networks there is no continuous flow of information between transmitter and receiver. The information is transmitted as bursts of frames that generally consist of four main fields: the preamble, the header, the payload and the trailer. The preamble is a sequence of ones and zeroes carrying information about the transmitter's timing in order to initially synchronize the receiver's clock, so that the receiver will be able to correctly achieve synchronization at bit and byte levels. The header includes information that is essential to the functionality of the wireless network protocol (eg. Destination and Source MAC addresses), while the payload contains the information that has to be transferred from one station to the other. In such a mobile network, the steering of the antenna must be performed during the preamble period and not later, since any loss of header information bits will result in the discarding of the whole frame.

Since this work focuses on the IEEE802.11 protocol [4], for the rest of this paper we will use the restrictions imposed by the IEEE802.11 protocol specifications. The standard frame of the IEEE802.11 protocol supports a 128-symbol preamble period for each transmitted frame [4]. This is translated to $128 \mu \mathrm{sec}$ of preamble time at $1 \mathrm{Mbps}$ and the received signal strength (RSS) of the incoming signal is calculated every 16 $\mu$ sec. Figure 1 shows that at this sampling rate and in order the receiver's circuits to be able to extract the synchronization information, the maximum number of samples of the RSS available for finding the direction of arrival of the incoming signal is 5 samples, or $80 \mu \mathrm{sec}$, which also determines the maximum duration for the convergence of the relative direction finding algorithm. This time window corresponds to the worst-case scenario, where the receiver does not detect an incoming signal during the first sample of the RSS, needs one sampling period in order to synchronize the internal clock for the RDF process and needs one more sampling period to set up for the Start Frame Delimiter (SDF) of the incoming header.

\section{A. DOA Techniques}

There are many proposals in the literature discussing how the directional beam of a mobile terminal, can be used to minimize the effect of multipath. Most of the proposed algorithms are targeting cell phone applications and they use phased arrays in the base station. These algorithms are inadequate for mobile computing applications, since they assume that there is a continuous flow of data on both directions of the communication link, and moreover they are based on the use of phased arrays that are hard to integrate in a mobile station, due to their cost and size.

Switched antenna arrays are more suitable for mobile computing applications. Switched arrays and antenna diversity schemes have already been integrated in wireless network protocols [5]. Signal evaluation algorithms that are governing these algorithms have been widely reported. Preston et al in [3] have proposed a very interesting method for acquiring DOA information using a switched beam array. All DOA finding methods are based on the following idea: the switched beam array produces $\mathrm{N}$ different radiation patterns, which divide the azimuth plane in $\mathrm{N}$ equal angular sectors. In order to find the DOA, the receiver scans all these patterns and measures the RSS of each sector. By comparing 
the different RSS of each sector, the receiver can determine the relative angle of arrival of the incident signal with varying accuracy, depending on the comparison method. Due to the fact that the computational burden of these methods is minimal, the time needed in order to compute the relative DOA is proportional to the total number $\mathrm{N}$ of the different sectors of the array.

\section{B. The Switched Beam Array}

The method proposed in this paper uses a switched beam array that produces omni-directional and directional beams. Such arrays have been recently reported in the literature and they are either patch [6], slot [7], or monopole arrays. In [7] we have proposed an active switched printed slot antenna array for the $5.2 \mathrm{GHz}$ band that may produce an omnidirectional pattern and many directional patterns in the azimuth, the narrower of which has a directivity of $8.56 \mathrm{dBi}$. Although this array was developed for the $5.2 \mathrm{GHz}$ frequency band, a scaled version of the array would be suitable for the $2.4 \mathrm{GHz}$ ISM frequency band of IEEE802.11, without affecting the radiation patterns reported in [7]. Figure 2 shows all the different radiation patterns that are adequate to cover all the azimuth angles by driving a different number of elements. It is evident that this array can produce an omnidirectional radiation pattern, it can divide the azimuth plane in a number of equal angular sectors $\left(\mathrm{N}_{1}=2\right)$, and finally each sector can be divided in a number of equal angular subsectors $\left(\mathrm{N}_{2}=3\right)$. These characteristics have a crucial impact in the implementation of the DOA determination method, as this is described in the next paragraph.

\section{The Relative Direction Finding Method}

We consider a switched beam array that can produce the following radiation patterns:

- An omni-directional pattern

- $\quad \mathrm{N}_{1}$ angular sectors of $\left(360 / \mathrm{N}_{1}\right)$-degrees

- $\mathrm{N}_{2}$ equal angular sub-sectors of $\left(\mathrm{N}_{1} / \mathrm{N}_{2}\right)$-degrees for each of the above $\mathrm{N}_{1}$ sectors,

or in the general case,

- $\quad \mathrm{N}_{\mathrm{k}}$ equal angular sub-sectors of $\left(\mathrm{N}_{\mathrm{k}-1} / \mathrm{N}_{\mathrm{k}}\right)$-degrees for each of the $\mathrm{N}_{\mathrm{k}-1}$ sub-sectors

When the receiver is in idle mode, the antenna is switched to the omni-directional pattern. Once the RSS rises above a certain threshold level, meaning that the antenna has received an incident signal, the procedure for determining the transmitter's relative direction begins. The receiver initially measures the RSS of all the $\mathrm{N}_{1}$ sectors. Once the sector with the best signal is found, then the receiver checks all the $\mathrm{N}_{2}$ sub-sectors of this sector. The algorithm continues
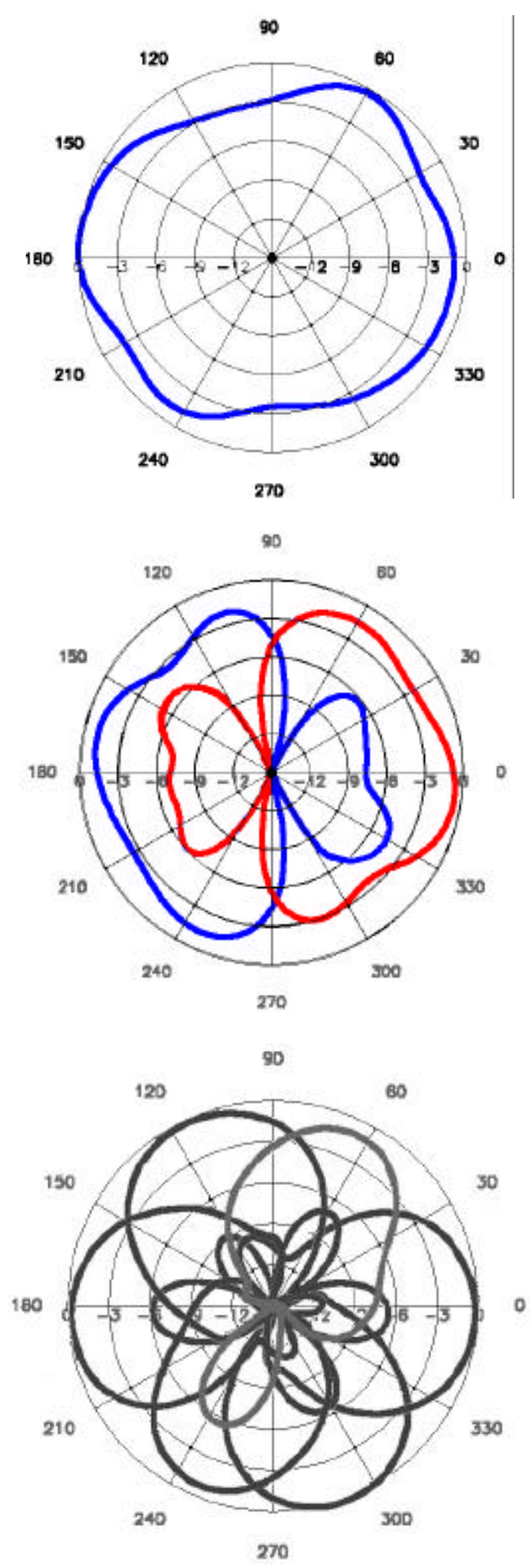

Fig. 2: Different switched array radiation patterns 
this tree-like search until the $\mathrm{N}_{\mathrm{k}}$ sector with the best performance is found. The accuracy of the DOA determination is equal to the beam-width of the $\mathrm{N}_{k}$ sectors. Following this procedure, the proposed RDF algorithm always finds the sector with the best performance, regardless of the number and the strength of the multipaths received by the antenna. In this version of the previously reported DOA determination method, the total time needed in order to compute the relative DOA is proportional to the sum:

$N=N_{1}+N_{2}+\ldots+N_{k}$

and not to the total number of narrow sub-sectors

$N=N_{1} \cdot N_{2} \cdot \ldots \cdot N_{k}$

Considering the switched beam array with the radiation patterns of Figure 2, $\mathrm{N}_{1}=2$ and $\mathrm{N}_{2}=3$. This means that the proposed direction finding technique needs 5 samples to determine the DOA of the incoming signal with an accuracy of 60 degrees, as opposed to the 6 samples needed for the previous version of the algorithm. It is evident that the proposed method is time efficient and robust and it is suitable to be implemented in IEEE802.11 wireless networks.

\section{LOCATION DETERMINATION}

In the previous section, we described a method for determining the DOA of an incoming signal, so that a station receiving a data frame can switch to a directional beam pointing at the LOS of the transmitter. In order to minimize the strong multipaths that arrive at the receiver with a small angular distance from the LOS, it is equally crucial to use a directional beam at the transmitter [1]. This can be achieved by using the previously mentioned DOA method and by associating certain MAC layer functions with different radiation patterns of the antenna used. The method is based on the development of a beam management function that communicates with the physical and the MAC layer entities and remains transparent to the functionality of these two layers. The MAC layer of the IEEE802.11 wireless protocol uses the CSMA/CA access algorithm and the proposed method can be applied to any wireless network that uses the same access mechanism.

\section{A. The CSMA/CA MAC layer}

The CSMA/CA protocol confronts the problem of collisions due to "hidden terminals" in the wireless networks. For example, as shown in Figure 3, station $\mathrm{C}$ will not sense that station $\mathrm{B}$ is transmitting a packet to station $\mathrm{A}$ and therefore,

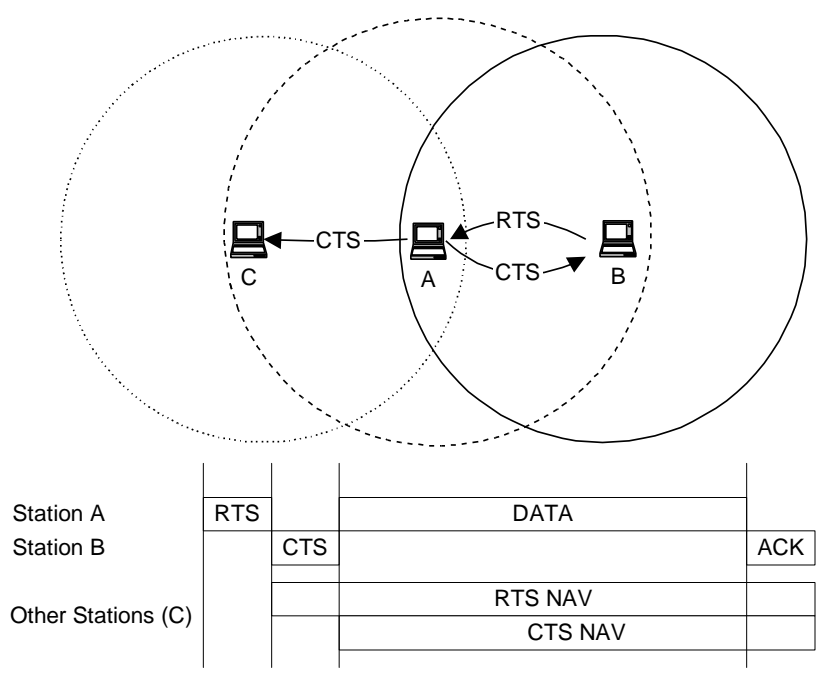

Fig. 3: The collision avoidance algorithm in IEEE802.11

it may also send a packet to station A, causing a collision that may not be detected by the transmitting stations. In order to reduce this probability the CSMA/CA defines the Virtual Carrier Sense mechanism that is described in the following paragraph.

A station having to transmit a packet, initially transmits a short control frame called Request-To-Send (RTS), which includes the source address, destination address and the duration of the transaction. The receiving station, whose MAC address equals the destination address, answers with a response control frame called Clear-to-Send (CTS), which includes the same duration information. All stations receiving either the RTS and/or the CTS, set their Virtual Carrier Sense indicator (called Network Allocation Vector NAV), and remain idle for the given duration. This mechanism reduces the collision probability by a station that is "hidden" from the transmitter, since the station hears the CTS and considers the medium as busy, until the end of the transaction. The duration information on the RTS also protects the transmitter from collisions during the acknowledgement frame (ACK) (from stations that are out of range of the acknowledging station). Fig. 4 shows a transaction between stations A and B and the NAV setting on their neighbors. The proposed method uses these control frames in order to determine the relative direction of other stations, without affecting the functionality of the CSMA/CA protocol.

\section{B. Beam Management Function}

The proposed direction determination method operates in a slow-time-varying environment, with a dominant LOS 
signal. The characteristics of the indoor channel, which are thoroughly described in [8], meet this specification. In such an environment, the channel characteristics do not change significantly over a period of some milliseconds, which corresponds to the duration of a packet exchange.

Figure 4 shows an example of using the proposed method for obtaining relative direction information on both sides of the communication link. Station A that has to transmit a packet to station B, uses the omni-directional pattern of its switched antenna array and sends an RTS frame.

All stations that "hear" the transmitter will initially receive the physical layer header of the packet. During the preamble period of this header, the DOA is determined, using the direction finding method described in the previous section. At the end of the RTS frame transmission, station B sends a CTS frame back to station A. During the preamble period of this frame, station A determines the relative direction of station B. At the end of RTS and CTS frames, all network contentions have been resolved, and the stations are free to use their directional beams for data transmission. Assuming that the indoor channel does not change significantly over the period of a CTS+DATA frame sequence, station A uses the directional beam that was chosen during the preamble period of the CTS frame and transmits the data frame. Station B uses the same direction finding process and by the end of the preamble period of the data frame, both the transmitter and the receiver know the relative direction of each other and direct their antenna beams to each other.
During the transmission of the ACK frame, station A may refresh the relative direction information of station $\mathrm{B}$.

Using the previously described relative direction determination method and assuming that the 2Mbps DSSS version of IEEE802.11 is used, the time $t_{f}$ required for station A to acquire relative direction information for station $B$ is $394 \mu \mathrm{sec}$. This information is not refreshed during the transmission of the data frame. During this period the channel does not change significantly [8] and the accuracy of the relative direction information does not deteriorate.

\section{Performance Results}

The proposed method was simulated using Matlab in order to study its effect on the IEEE802.11 wireless protocol performance. The wireless link and the antennas were modeled as described in [9]. The channel performance was estimated for all possible combinations of transmitting and receiving antenna beams. Due to the bi-directionality of the wireless channel, the following cases are considered:

- Omni-directional to Omni-directional (OO)

- Omni-directional to Directional (OD)

- Directional to Directional (DD)

From channel's statistical analysis we found the delay spread of the radio channel for these cases, which is $68 \mathrm{nsec}$ for the OO case, $36 \mathrm{nsec}$ for the OD case, and $23 \mathrm{nsec}$ for the DD case. Using the delay spread of the channel, we were able to

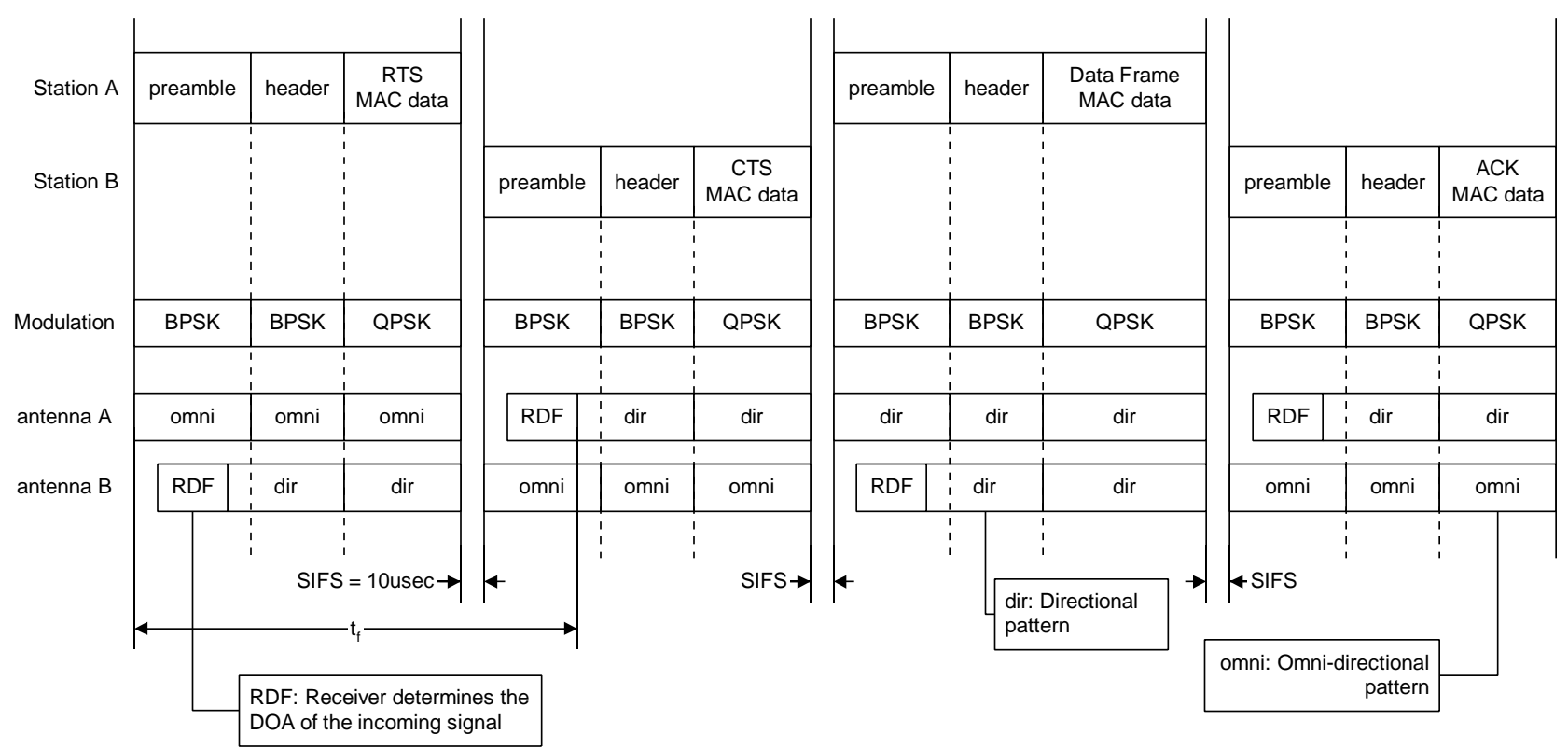

Fig. 4: The proposed delative direction determination method 


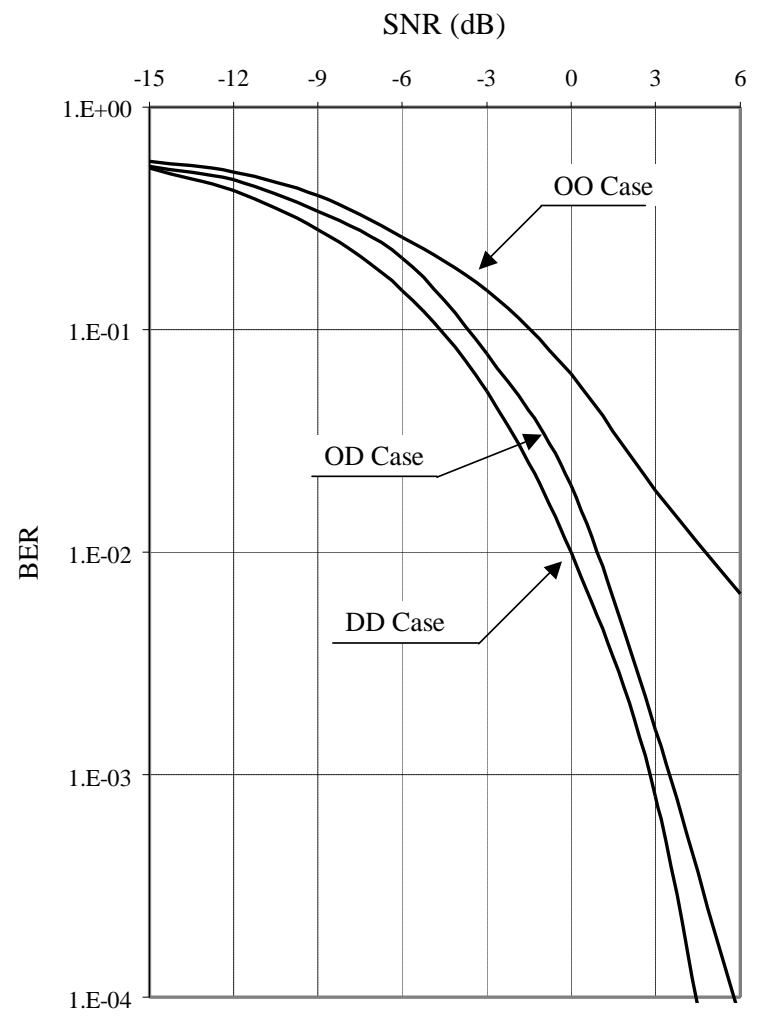

Fig. 5: BER chart of the BPSK DSSS modulation

compute the bit error rate (BER) of the wireless transmission. The IEEE802.11 protocol uses two modulation schemes at 2Mbps: BPSK for the physical layer header of each frame, and QPSK for the MAC layer frame. The BER of the transmission for the three antenna beam combinations and the two modulation schemes is shown in Figures 5 and 6 respectively. It is evident that the best performance of the wireless link is achieved when the antennas on both ends of the link are switched to their directional beams and when both are oriented to aim each other.

\section{CONCLUSIONS}

We presented a relative direction determination method for IEEE802.11 mobile computing networks. The method is based on the use of a switched beam array with variable diversity gain and a RDF algorithm that can be efficiently used in wireless data networks. The proposed method exploits the RTS and CTS frames used in IEEE802.11 in order to acquire relative direction information on both ends of the communication link, without affecting the MAC layer functionality and without requiring any additional bandwidth. As the simulation results show, the proposed method of relative direction determination improves the overall network performance.

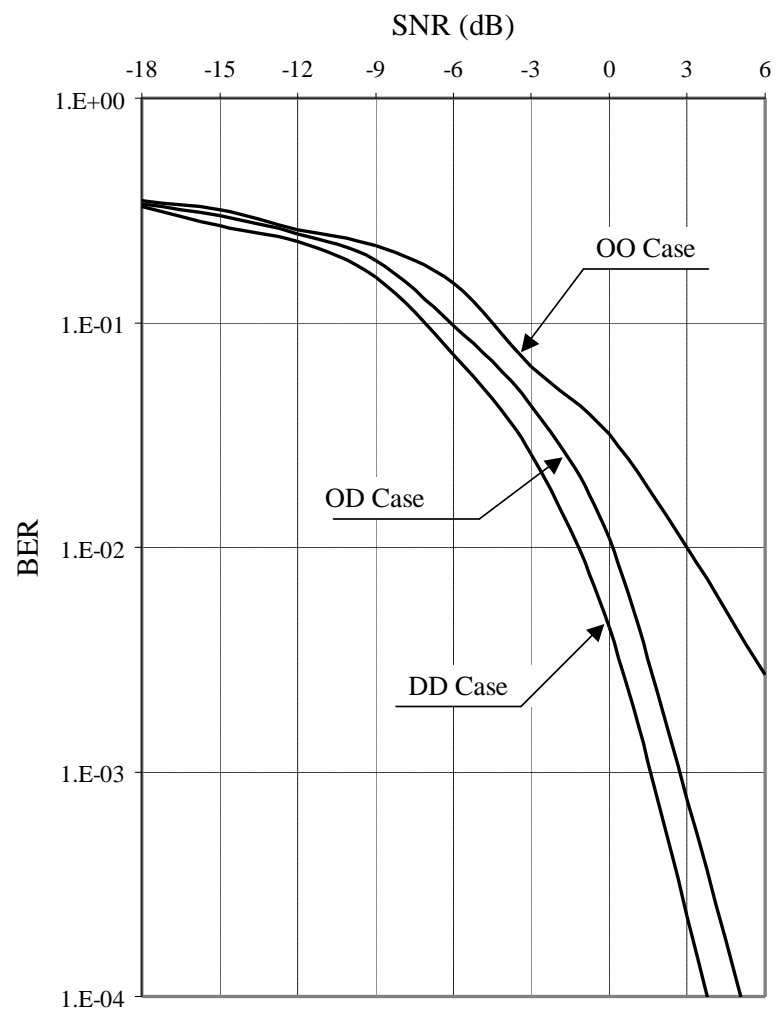

Fig. 6: BER chart of the QPSK DSSS modulation

\section{REFERENCES}

[1] P. Driessen, "Gigabit/s Indoor Wireless Systems with directional antennas", IEEE Transactions on Communications, Vol.44, No.8, August 1996, p.p. 1034-1043.

[2] P. Lehne, M. Pettersen, "An overview of smart antenna technology for mobile communications systems", IEEE Communications Surveys, http://www.comsoc.org/ magazines/surveys/4q99issue/lehne.html, Fourth Quarter 1999, Vol. 2, No. 4.

[3] S. Preston, V. Thiel, T. Smith, S. O' Keefe, J. Lu, "Base-station tracking in mobile communications using a switched parasitic antenna array", IEEE Transactions on Antennas \& Propagation, Vol.46, No.6, June 1998, p.p. 841-844.

[4] INTERNATIONAL STANDARD ISO/IEC 8802-11 ANSI/IEEE Std 802.11 First edition 1999-00-00 Part 11: Wireless LAN Medium Access Control (MAC) and Physical Layer (PHY) specifications. ISBN 0-7381$1658-0$.

[5] PRISM® chipset, Intersil Corp., http://www.intersil.com

[6] H. Iwasaki "Slot coupled back-to-back microstrip antenna with an omnior a bi-directional radiation pattern", IEE Proceedings-Microwave, Antennas and Propagation., Vol.46, No.3, June 1999, p.p. 219-223.

[7] A. Kalis, Th. Antonakopoulos, V. Makios, "A printed circuit switched array antenna for indoor communications", IEEE Transactions on Consumer Electronics, Vol.46, No.3, August 2000, p.p. 531-538.

[8] A. Saleh, R. Valenzuela, "A statistical model for indoor multipath propagation", IEEE Journal on Selected Areas in Communications, Vol. SAC-5, No.2, Feb. 1987, pp. 128-137.

[9] T. Zwick, C. Fisher, D. Didaskalou, W. Wiesbeck, "A Stochastic Spatial Channel Model Based on Wave-Propagation Modeling", IEEE Journal on Selected Areas in Communications, Vol. 18, No. 1, January 2000, pp 6-15. 